\title{
Transglycosylated starch accelerated intestinal transit and enhanced bacterial fermentation in the large intestine using a pig model
}

\author{
Barbara U. Metzler-Zebeli ${ }^{1 *}$, Monica A. Newman ${ }^{1}$, Andrea Ladinig ${ }^{2}$, Wolfgang Kandler ${ }^{3}$, Dietmar Grüll ${ }^{4}$ and \\ Qendrim Zebeli ${ }^{1}$ \\ ${ }^{1}$ Institute of Animal Nutrition and Functional Plant Compounds, University of Veterinary Medicine Vienna, 1210 Vienna, \\ Austria \\ ${ }^{2}$ University Clinic for Swine, Department for Farm Animals and Veterinary Public Health, University of Veterinary Medicine \\ Vienna, 1210 Vienna, Austria \\ ${ }^{3}$ University of Natural Resources and Life Sciences, Vienna, Department of Agrobiotechnology, IFA-Tulln, 3430 Tulln, Austria \\ ${ }^{4}$ Agrana Research and Innovation Center GmbH, 3430 Tulln, Austria \\ (Submitted 14 January 2019 - Final revision received 22 March 2019 - Accepted 25 March 2019 - First published online 20 June 2019)
}

\section{Abstract}

Resistant starch can alter the intestinal nutrient availability and bulk of digesta, thereby modulating the substrate available for microbial metabolic activity along the gastrointestinal tract. This study elucidated the effect of transglycosylated starch (TGS) on the retention of digesta in the upper digestive tract, ileal flow and hindgut disappearance of nutrients, and subsequent bacterial profiles in pigs. Fourteen ileal-cannulated growing pigs were fed either the TGS or control (CON) diet in a complete crossover design. Each period consisted of a 10-d adaptation to the diets, followed by 3-d collection of faeces and ileal digesta. Consumption of TGS decreased the retention of digesta in the stomach and small intestine, and increased ileal DM, starch, Ca and P flow, leading to enhanced starch fermentation in the hindgut compared with CON-fed pigs. TGS increased ileal and faecal total SCFA, especially ileal and faecal acetate and faecal butyrate. Gastric retention time positively correlated to Klebsiella, which benefitted together with Selenomonas, Lactobacillus, Mitsuokella and Coriobacteriaceae from TGS feeding and ileal starch flow. Similar relationships existed in faeces with Coriobacteriaceae, Veillonellaceae and Megasphaera benefitting most, either directly or indirectly via cross-feeding, from TGS residuals in faeces. TGS, in turn, depressed genera within Ruminococcaceae, Clostridiales and Christensenellaceae compared with the CON diet. The present results demonstrated distinct ileal and faecal bacterial community and metabolite profiles in CON- and TGS-fed pigs, which were modulated by the type of starch, intestinal substrate flow and retention of digesta in the upper digestive tract.

Key words: Resistant starch type 4: Pigs: Intestinal nutrient flow: Bacterial microbiota: Starch digestibility

Dietary fibres are components of different physicochemical and rheological properties. Consequently, the various kinds of fibres differently affect the intestinal physiology and microbiota ${ }^{(1)}$. The health benefits of resistant starch (RS), one type of dietary fibre, have been linked to improved glycaemia, insulinaemia and lipidaemia and by acting as a prebiotic, thereby improving satiety and bowel health ${ }^{(2,3)}$. The benefits of RS can be related to a lower glucose release in the small intestine ${ }^{(3)}$ and its stimulating effect on intestinal SCFA produced during the fermentation of $\mathrm{RS}^{(2,4)}$. The SCFA enhance the secretion of incretins, which modulate the postprandial insulin response and inhibit proinflammatory signalling cascades via G-protein receptor activation and signalling ${ }^{(2,4)}$.

Dietary fibres that produce intraluminal viscosity can delay gastric emptying, as well as inhibit the rate of digestion and absorption of nutrients ${ }^{(3,5)}$. For this reason, the effect of RS to increase the bulk of digesta has been often investigated in regard to the satiety feeling after a meal ${ }^{(6)}$. Besides, delayed stomach emptying and slower transit may enhance microbial activity in the upper digestive tract. Although RS effects on postprandial changes in digestion, metabolic and hormonal profiles, and intestinal SCFA production have been repeatedly characterised $^{(6,7)}$, little quantitative information is available on the effects of RS-related alterations in the kinetics of digestion and transit on the microbial community in the gastrointestinal tract.

Aside from RS2 (high-amylose starch) and RS3 (retrograded starch), the interest in RS4 has risen ${ }^{(3)}$. RS4 are chemically modified starches and, as such, may replace rapidly digestible modified starches in processed foods. In that way, RS4 may fulfil dual

Abbreviations: CON, control; DA, discriminant analysis; RS, resistant starch; sPLS, sparse partial least squares; TGS, transglycosylated starch.

* Corresponding author: B. U. Metzler-Zebeli, email barbara.metzler@vetmeduni.ac.at 
functionality by improving texture and rheological properties of the food product and increasing the daily consumption of dietary fibre. In general, starches can be chemically modified in different ways, including transglycosylation, esterification or crosslinking ${ }^{(3)}$. The physiological effects will depend on the respective RS4 and differ from other types of RS, such as high-amylose starch (RS2) or retrograded starch (RS3) ${ }^{(3)}$, necessitating to investigate the potential health benefits of each RS4 individually. While decreasing the postprandial insulin response and altering the serum lipid metabolome profiles ${ }^{(8)}$, transglycosylated starch (TGS) altered the gastric, ileal, caecal and colonic bacterial microbiomes in growing pigs ${ }^{(9)}$. Notably, the qualitative bacterial changes were almost identical in the various gastrointestinal segments, whereas the TGS effects on SCFA were the greatest in the proximal and mid-colon ${ }^{(9)}$. In order to understand the effect of TGS on nutrient flows and microbial substrate availability, we investigated the effect of TGS consumption on the rate of passage of digesta in the upper digestive tract and quantified ileal flow and hindgut disappearance of nutrients using the pig model. In determining ileal and faecal bacterial microbiome and SCFA, we further assessed the dependencies between nutrient availability and alterations in bacterial composition and fermentation metabolite profiles in ileal digesta and faeces. We hypothesised that, due to an increased digesta bulk caused by the largely reduced small intestinal starch digestion, TGS would slow down the transit in the upper digestive tract, leading to an enhanced and prolonged nutrient flow to the large intestine. We further assumed that we could identify bacterial taxa having an association with the intestinal transit and nutrient flow. We used the pig model due to the high similarity in digestive physiology and metabolic responses to humans ${ }^{(10,11)}$. Nevertheless, pigs and humans differ in their microbial colonisation, and pigs have a shorter retention of digesta in the various segments of the gastrointestinal tract compared with humans ${ }^{(12)}$. The advantage of using the ileal-cannulated pig is that this animal model allows the collection of ileal digesta samples from healthy individuals, which is hardly possible from healthy human volunteers.

\section{Materials and methods}

\section{Ethical approval}

All procedures involving animal handling and treatment were approved by the institutional ethics committee of the University of Veterinary Medicine Vienna and the national authority according to paragraph 26 of Law for Animal Experiments, Tierversuchsgesetz - TVG (GZ BMWF-68.205/ 0051-II/3b/2013).

\section{Animals}

Seven castrated male pigs (Large White; initial body weight 54.8 (sD 3.4) kg; age 3-4 months) were obtained from the university research pig farm (University of Veterinary Medicine Vienna) 1 week prior to ileal cannulation. Pigs were housed in individual metabolism pens $(1.0 \times 1.2 \mathrm{~m})$ with Plexiglass walls for visual contact in an environmentally controlled room $\left(21 \pm 1^{\circ} \mathrm{C}\right)^{(13)}$. Each pen was equipped with a single-space feeder and a nipple drinker for ad libitum access to demineralised water. Pens were cleaned daily with water and faeces were removed several times daily. Health status of pigs was monitored daily throughout the experiment. Pigs were surgically fitted with a simple T-cannula (tubus $9 \mathrm{~cm}$, foot $9 \mathrm{~cm}$, inner diameter $2 \cdot 0 \mathrm{~cm}$, outer diameter $2.3 \mathrm{~cm}$; LKT - Laboratorium für Kunststofftechnik GmbH), inserted at the distal ileum to allow for the collection of ileal digesta ${ }^{(13)}$. Postsurgery, the skin around the cannula was cleaned with lukewarm water at least twice daily, treated with a skin-protecting paste (Stomahesive Paste, Convatec), and foam material was placed between the retaining ring and the skin to absorb any leaking digesta to prevent erythema ${ }^{(14)}$. Post-surgery, pigs were allowed a 9-d recovery period, where feed amounts were gradually increased until reaching pre-surgery levels. Upon completion of the experiment, pigs were sedated (Narketan, $10 \mathrm{ml} / \mathrm{kg}$ body weight (Ketamine $\mathrm{HCl}$; Vétoquinol AG) and Stresnil, $3 \mathrm{ml} / \mathrm{kg}$ body weight (Azaperone; Biokema SA)) and euthanised by intracardiac injection of T61 $(10 \mathrm{ml} / \mathrm{kg}$; Embutramide; MSD Animal Health).

\section{Experimental design, diets and feeding}

Prior to the start of the experiment, pigs were fed a commercial cereal-based grower diet (metabolisable energy $13.4 \mathrm{MJ} / \mathrm{kg}$; crude protein $16.8 \%$ as-fed basis) ${ }^{(9)}$. Following recovery, pigs were randomly allotted to one of two dietary treatments according to a complete crossover design with two 16-d replicate periods (online Supplementary Fig. S1). This provided a total of seven observations per dietary treatment. Each replicate period consisted of $10-\mathrm{d}$ adaptation to diets, followed by 3 -d collection of faeces and 3-d collection of ileal digesta. The advantage of using a crossover design is that it requires a smaller sample size than a parallel design, without compromising the level of statistical power and precision, which is helpful when using an ileal cannulation model. Nevertheless, it may be advisable to include a washout period in order to avoid carryover effects from one to the other experimental period, especially as the effect of short-term feeding of TGS on the intestinal passage rate, nutrient flow and microbiota composition was investigated in the present study. We did not include a washout period due to the increasing risk of growth-related losses of cannulae with progressing time from the surgery, and maturational changes in the intestinal microbiota with increasing age of the pig. In order to balance for simple first-order carryover effects of one treatment into the following treatment period, the diets were randomised among pigs in both replicate batches.

The two experimental diets were formulated to meet or exceed current recommendations for nutrient requirements of growing pigs ${ }^{(15)}$ and only differed in the starch component (Table 1). The starch component of the control (CON) diet was a rapidly digestible waxy maize starch (Agrana Research and Innovation Center GmbH), whereas in the TGS diet, $50 \%$ of the native waxy maize starch was replaced by TGS (Agrana Research and Innovation Center $\mathrm{GmbH})^{(9,16)}$. As described before ${ }^{(9,16)}$, acid-catalysed transglycosylation of the native waxy maize starch was used to produce TGS. This process rearranged the glycosidic bonds in the native waxy maize starch, resulting in 
Table 1. Ingredients and chemical composition of the control (CON) diet and transglycosylated starch (TGS) diet

\begin{tabular}{|c|c|c|}
\hline & CON & TGS \\
\hline \multicolumn{3}{|c|}{ Ingredients (g/kg, as-fed basis) } \\
\hline Waxy maize starch* & $721 \cdot 0$ & 360.5 \\
\hline TGS maize starch* & 0 & 360.5 \\
\hline Casein & $180 \cdot 0$ & $180 \cdot 0$ \\
\hline Lignocellulose $\dagger$ & $40 \cdot 0$ & $40 \cdot 0$ \\
\hline Monocalcium phosphate & $40 \cdot 0$ & $40 \cdot 0$ \\
\hline Rapeseed oil & $10 \cdot 0$ & $10 \cdot 0$ \\
\hline Vitamin-mineral premix $\ddagger$ & $6 \cdot 0$ & $6 \cdot 0$ \\
\hline Titanium dioxide & $3 \cdot 0$ & 3.0 \\
\hline \multicolumn{3}{|c|}{ Analysed chemical composition } \\
\hline DM $(\mathrm{g} / \mathrm{kg})$ & 941 & 947 \\
\hline Crude protein (g/kg DM) & 169 & 170 \\
\hline Crude ash $(\mathrm{g} / \mathrm{kg})$ & $52 \cdot 1$ & $46 \cdot 2$ \\
\hline Total starch $(\mathrm{g} / \mathrm{kg} \mathrm{DM}) \S$ & $72 \cdot 8$ & 690 \\
\hline $\mathrm{Ca}(\mathrm{g} / \mathrm{kg} \mathrm{DM})$ & $7 \cdot 4$ & $7 \cdot 6$ \\
\hline$P(g / k g D M)$ & 4.5 & 4.6 \\
\hline Gross energy $(\mathrm{MJ} / \mathrm{kg})$ & $17 \cdot 6$ & 17.9 \\
\hline
\end{tabular}

${ }^{*}$ Agrana Research and Innovation Center $\mathrm{GmbH}$.

† FibreCell (Agromed Austria GmbH).

‡ Provided per $\mathrm{kg}$ of complete diet (GARANT GmbH): $4800 \mu \mathrm{g}$ of vitamin A, $50 \mu \mathrm{g}$ of vitamin $D_{3}, 125 \mathrm{mg}$ of vitamin $E, 2.0 \mathrm{mg}$ of vitamin $B_{1}, 6.0 \mathrm{mg}$ of vitamin $B_{2}, 3.0 \mathrm{mg}$ of vitamin $B_{6}, 0.03 \mathrm{mg}$ of vitamin $B_{12}, 3.0 \mathrm{mg}$ of vitamin $K_{3}, 30 \mathrm{mg}$ of niacin, $15.0 \mathrm{mg}$ of pantothenic acid, $900 \mathrm{mg}$ of choline chloride, $0.15 \mathrm{mg}$ of biotin, $1.5 \mathrm{mg}$ of folic acid, $200 \mathrm{mg}$ of vitamin C, $4.6 \mathrm{~g}$ of Ca, $2.3 \mathrm{~g}$ as digestible $\mathrm{P}, 2.4 \mathrm{~g}$ as Na, $2.0 \mathrm{~g}$ of Cl, $3.2 \mathrm{~g} \mathrm{~K}$, $1.0 \mathrm{~g} \mathrm{Mg}, 50 \mathrm{mg}$ of $\mathrm{Mn}$ (as $\mathrm{MnO}$ ), $100 \mathrm{mg}$ of $\mathrm{Zn}\left(\mathrm{as} \mathrm{ZnSO}_{4}\right), 120 \mathrm{mg}$ of Fe (as FeSO $\mathrm{F}_{4}$ ), $15.6 \mathrm{mg}$ of $\mathrm{Cu}\left(\right.$ as $\left.\mathrm{CuSO}_{4}\right), 0.5 \mathrm{mg}$ of $\mathrm{Se}\left(\right.$ as $\left.\mathrm{Na}_{2} \mathrm{SeO}_{3}\right), 1.9 \mathrm{mg}$ of iodine (as $\left.\mathrm{Ca}\left(\mathrm{IO}_{3}\right)_{2}\right)$.

$\S$ Total starch in the CON diet determined by enzymatic spectrophotometric assay (R-Biopharm), and in the TGS diet by perchloric acid treatment of samples and measurement of resulting glucose using $\mathrm{HPLC}^{(15)}$.

eight types of glycosydic bonds: $\alpha(1,2), \alpha(1,3), \alpha(1,4), \alpha(1,6)$, $\beta(1,2), \beta(1,3), \beta(1,4)$, and $\beta(1,6)$-glycosidic bonds and a total dietary fibre content of $50 \%$ in the final TGS product (method $2009.01)^{(17)}$. Daily feed allowances were estimated to correspond to approximately three times the estimated energy required for maintenance ${ }^{(15)}$, which were divided into two equal meals that were fed at 08.00 and 16.00 hours as a mash and mixed with water at a ratio of about 2:1. Potential feed spillage and leftovers in feeding bowls were collected after feeding

\section{Sample collection and digesta flow marker application}

In each of the two experimental periods, fresh faecal samples were collected from slatted flooring and tray beneath the cage on experimental days 11-13 (online Supplementary Fig. S1) via grab sampling ${ }^{(13)}$. Subsamples of freshly defecated faeces were immediately frozen at $-80^{\circ} \mathrm{C}$ for the analysis of faecal microbiota and at $-20^{\circ} \mathrm{C}$ for SCFA. The remaining faecal samples were stored at $-20^{\circ} \mathrm{C}$ for proximate nutrient analysis. Ileal digesta samples were collected on days 14 and 15 between 08.00 and 18.00 hours into $10 \times 4 \mathrm{~cm}$ plastic bags that were attached to the barrel of each cannula using rubber bands. Each bag contained $4 \mathrm{ml}$ of $2.5 \mathrm{~m}$ formic acid to minimise bacterial fermentation. Full bags were removed, or at least every 30 min. Ileal digesta samples were collected without formic acid for bacterial microbiota, SCFA and lactate analyses at 11.00 and 14.00 hours. Subsamples for bacterial microbiota were immediately frozen at $-80^{\circ} \mathrm{C}$, and subsamples for SCFA and lactate were stored at $4^{\circ} \mathrm{C}$ until digesta samples from both time-points were obtained. Then, digesta was pooled by pig, homogenised and frozen at $-20^{\circ} \mathrm{C}$ for later analysis. On day 16 of each experimental run, $40 \mathrm{ml}$ of liquid marker (chromium-ethylenediaminetetraacetic acid, Cr-EDTA) and $1 \mathrm{~g}$ of solid marker $\left(\mathrm{Yb}_{2} \mathrm{O}_{3}\right)$ were mixed into the morning meal ${ }^{(13)}$. Ileal digesta samples were collected postprandially at 0, 30, 90, 120, 180, 240, 300, 360, 420 and $480 \mathrm{~min}$. As ileal digesta flow can be irregular, the actual collection times were recorded.

\section{Chemical analysis}

Prior to chemical analyses, ileal and faecal subsamples for proximate nutrient analysis were lyophilised (Gamma 2-20; Martin Christ Gefriertrocknungsanlagen $\mathrm{GmbH}$ ). Ileal, faecal and diet samples were homogenised and ground through a 0.5-mm screen (GRINDOMIX GM200; Retsch GmbH). Diets and freeze-dried ileal digesta and faeces were analysed for $\mathrm{DM}$, crude ash, crude protein $(\mathrm{N} \times 6.25$ by the Kjeldahl method), Ca and $\mathrm{P}^{(18)}$. Gross energy of diets, digesta and faeces was measured using an isoperibolic bomb calorimeter (C200; IKA-Werke $\mathrm{GmbH}$ and Co. KG), with benzoic acid as calibration standard ${ }^{(18)}$. The total starch content of the CON diet was determined via the UV method (Enzymatic BioAnalysis; R-Biopharm) using a spectrophotometer (DR 2800; Hach Lange $\mathrm{GmbH}$ ), whereas to measure total starch content of the TGS diet, soluble TGS was leached out of the samples and treated with perchloric acid ${ }^{(8)}$. Thereafter, the resulting glucose was measured using HPLC (Ultimate 3000; Thermo Fisher Scientific) equipped with an Aminex HPX-87H separation column and a Refratomax 520 detector. The starch content in ileal digesta and faeces was measured accordingly. Titanium dioxide was measured spectrophotometrically (Hitachi U-3000; Metrohm INULA GmbH) at $405 \mathrm{~nm}$ in feed, faeces and ileal digesta after Kjeldahl extraction ${ }^{(19)}$, whereas commercially available kits (Megazyme K-DLATE) were used to determine $\mathrm{D}$ - and L-lactate concentrations in fresh ileal digesta and faecal samples. Concentrations of SCFA in fresh ileal digesta and faecal samples were analysed using gas chromatography ${ }^{(13)}$. The $\mathrm{pH}$ in fresh ileal digesta and faeces was determined using a Beckman $\Phi 63$ pH meter (Beckman Coulter).

\section{Passage rate marker analysis}

Inductively coupled plasma mass spectrometry was applied to determine $\mathrm{Cr}$ and $\mathrm{Yb}$ as previously described ${ }^{(13)}$. The measurements were performed on a double-focusing sector field instrument (Finnigan ELEMENT2; Thermo Electron Corporation) equipped with a CETAC ASX-520 autosampler (CETAC Technologies) using $50 \mathrm{mg}$ of lyophilised ileal digesta. The digesta were weighed into $10 \mathrm{ml}$ glass tubes and digested with $2 \mathrm{ml} 65 \%$ nitric acid $\left(60 \mathrm{~min}\right.$ at $\left.95^{\circ} \mathrm{C}\right)$. Thereafter, the solutions were transferred into clean and pre-weighted $50 \mathrm{ml}$ tubes of polypropylene, filled up to $50 \mathrm{~g}$ on a laboratory balance and shaken. After sedimentation, the solutions were diluted 1:100 with $0.5 \%(\mathrm{v} / \mathrm{v})$ nitric acid, and $20 \mu \mathrm{g} / \mathrm{l} \mathrm{Sc}, 10 \mu \mathrm{g} / \mathrm{l} \mathrm{In}$ and $10 \mu \mathrm{g} / \mathrm{l} \mathrm{Tl}$ were added as internal standards. The following 
nuclides were measured in medium-resolution mode, $\mathrm{R}_{\mathrm{s}}=4000,10 \%$ valley definition ${ }^{31} \mathrm{P},{ }^{44} \mathrm{Ca},{ }^{45} \mathrm{Sc},{ }^{52} \mathrm{Cr},{ }^{173} \mathrm{Yb}$ and ${ }^{205} \mathrm{Tl}$. Quantitative analysis of the samples was performed by external calibration.

The excretion curves of ileal markers, including $\mathrm{Yb}_{2} \mathrm{O}_{3}$ as a solid marker and Cr-EDTA as a liquid marker, were fitted to a two-compartment kinetics model, implemented in the NLIN procedure (iterative Marquardt method) of SAS (version 9.4, SAS Institute. Inc.) as described before ${ }^{(13,20)}$. These models estimate the fractional passage rates from two compartments $\left(\operatorname{slow}\left(\mathrm{k}_{\mathrm{S}}\right)\right.$ and fast $\left(\mathrm{k}_{\mathrm{F}}\right)$, where $\mathrm{k}_{\mathrm{S}}$ represents passage out of the stomach; and $\mathrm{k}_{\mathrm{F}}$, passage out of the small intestine), including a time delay. The time delay indicates the time lapse between pulse dosing and the first appearance of markers in ileal digesta (or transit time due to displacement flow of digesta, in minutes). The time lapse between pulse dosing and the reach of peak ileal marker excretion was considered as the time of peak flow (minutes). Maximum total mean retention time (TMRT) was calculated as the sum of maximum mean retention times in the stomach $\left(\right.$ GMRT $\left.=1 / \mathrm{k}_{\mathrm{S}}\right)$ and small intestine $\left(\mathrm{SMRT}=1 / \mathrm{k}_{\mathrm{F}}\right)$, including the time delay.

\section{DNA isolation, Illumina MiSeq sequencing and bioinformatics analysis}

The PowerSoil DNA isolation kit (MoBio Laboratories) was used to extract total DNA from $250 \mathrm{mg}$ of the homogenised ileal digesta and faecal samples. Modifications included an additional heating step $\left(70^{\circ} \mathrm{C}\right.$ for $\left.10 \mathrm{~min}\right)$ between mixing the digesta samples with $\mathrm{C} 1$ buffer and bead beating for proper lysis of bacteria $^{(21)}$. DNA concentration was measured using the Qubit double-stranded DNA HS assay kit (Life Technologies) on the Qubit 2.0 fluorometer (Life Technologies). The 16S rRNA gene PCRs, library preparation and sequencing on an Illumina MiSeq sequencing v2 platform (Illumina Inc.) were performed with Microsynth as described previously ${ }^{(9)}$. The V3-V5 hypervariable regions of bacterial 16S rRNA genes were amplified using the primers 357F-HMP (5'-CCTACGGGAG GCAGCAG-3') and 926R-HMP (5'-CCGTCAATTCMTTT RAGT-3') to produce an amplicon size of approximately 523 bp (Peterson et al., 2009). Libraries were constructed by ligating sequencing adapters and indices onto purified PCR products using the Nextera XT sample preparation kit (Illumina Inc.). Equimolar quantities of each library were pooled and sequenced using a 300-bp read length paired-end protocol. After sequencing, the overlapping paired-end reads were de-multiplexed, trimmed of Illumina adaptor residuals using cutadapt (https://cutadapt. readthedocs.io/en/stable/) and stitched using USEARCH (drive $5 / \mathrm{com}$ ) with Microsynth.

The merged FASTQ sequences were analysed using QIIME (Quantitative Insights Into Microbial Ecology) software package, version $1.9 .1^{(22)}$. After quality trimming of stitched reads using a quality threshold of $q>20$, the UCHIME method using the 64-bit version of USEARCH (drive 5.com) ${ }^{(23,24)}$ and the GOLD database (drive5.com) were used to screen for and exclude chimeric sequences. Open-reference operational taxonomic unit (OTU) picking was performed at $97 \%$ similarity level using UCLUST $^{(23)}$, and taxonomy was assigned against the
Greengenes database (gg_13_8; http://qiime.org/home_static/ dataFiles.html). Rare OTUs with less than ten sequences were removed. The raw sequence reads were uploaded to the NCBI Bioproject databank (PRJNA514964).

Differences in hit count abundances of bacterial genera were assessed using the R package 'DESeq2' (version 1.14.1) ${ }^{(25,26)}$. The 'DESeq' function within the DESeq2 package was used to test for differentially abundant taxa by dietary starch. In using this function, data were normalised to the size factors of the libraries and dispersion estimation. Specifically, this function models raw counts using a negative binomial distribution and adjusts internally for 'size factors' that normalise for differences in sequencing depth between sample libraries. We also pre-filtered the dataset to keep only features that have at least ten reads total using the $\mathrm{R}$ command in DESeq2 rowSums(counts (deseq_data)) $\geq 10$ ' to remove low-count taxa. DESeq2 default settings were used to replace and filter for count outliers. Differential taxa abundance between treatments was identified using the 'Wald' test ${ }^{(25)}$. Data were listed as normalised read counts per feature. The correction of $P$ values relating to the taxonomic profiles was performed using the BenjaminiHochberg false discovery rate (FDR ${ }^{(27)}$. To account for multiple comparisons, we considered a type I error rate of $\leq 0.05$ and a FDR-adjusted $P$ value ( $q$ value) $\leq 0 \cdot 10$ as significant. Mean counts for each dietary starch were computed using the DESeq function within the DESeq2 package. Statistical assessment of dissimilarity matrices (Bray-Curtis) derived from ileal and faecal OTU data was performed using the 'adonis2' function (PERMANOVA) and visualised in two-dimensional nonmetric multidimensional scaling (NMDS) ordination plots obtained with the 'metaMDS' function in the vegan $\mathrm{R}$ package (version 2.5.2) ${ }^{(28)}$. Alpha diversity measurements were also performed by means of the vegan $\mathrm{R}$ package.

In addition, sparse partial least squares (sPLS) and relevance network analysis were performed using the package 'mixOmics' in $\mathrm{R}^{(29,30)}$ to integrate data of genera (0.01\% of all reads) and fermentation metabolites, including digesta $\mathrm{DM}$ content and $\mathrm{pH}$ with nutrient flow and digesta retention parameters in ileal and faecal samples, in order to identify dependencies among the data. The 'network' function calculated a similarity measure between $X$ and $Y$ variables in a pairwise manner. In the graphs, each $X$ and $Y$ variable corresponds to a node, whereas the edges display the associations between the nodes. The size of the nodes is arbitrary, depending on the name of the variable.

In order to further understand ileal and faecal networking, multi-group supervised DIABLO N-integration was performed to identify the key features and their relations among each other, also by means of the package 'mixOmics' (version 6.3) ${ }^{(30)}$ in R studio (version 1.1.456). Supervised multi-group sPLS-discriminant analysis (sPLS-DA) was used to integrate the datasets of relative abundances of genera, fermentation metabolites including digesta $\mathrm{DM}$ content and $\mathrm{pH}$, and nutrient flow in order to classify and select key features from each dataset. Tuning of sPLS-DA parameters was performed using the 'tune' function to identify the most influential genera, fermentation metabolites and nutrients in digesta or faeces with the lowest possible error rate - finally selecting ten genera, five fermentation metabolites and four nutrient genes each for components 1 and 2, 
respectively. In doing so, the 'tune' function returns a set of 'keepX' values for each dataset that shows the best predictive performance for all the components in the model, while simultaneously performing repeated leave-one-out crossvalidation using the function 'nrepeat'(30). The assessment of the performance of the final model by cross-validation showed the lowest possible error rate of, on average, 35 and $27 \%$ for components 1 and 2, respectively, for ileal datasets. For ileal datasets, cross-validation of the final model revealed the lowest possible error rate of, on average, 5.4 and $3.1 \%$ for components 1 and 2, respectively.

\section{Calculations and statistical analysis}

The amounts of DM protein, ash, starch ( $\mathrm{g} / \mathrm{kg}$ DM intake) and gross energy (MJ/kg DM intake) remaining at the distal ileum and excreted in faeces and post-ileal disappearance were calculated as previously reported ${ }^{(13)}$ :

Amount remaining at the terminal ileum or faeces ( $g$ or $\mathrm{MJ} / \mathrm{kg}$ $\mathrm{DM}$ intake $)=($ concentration of dietary constituent in digesta $/$ faeces $\times\left(\mathrm{TiO}_{2}\right.$ in diet $/ \mathrm{TiO}_{2}$ in digesta/faeces $\left.)\right)$

Post-ileal disappearance $(\mathrm{g}$ or $\mathrm{MJ} / \mathrm{kg} \mathrm{DM}$ intake)=amount remaining at terminal ileum - amount excreted in faeces

A power test analysis estimated according to Kononoff \& Hanford ${ }^{(31)}$ and based on previous data for similar variables, such as intestinal microbiota composition and digestibility ${ }^{(13,32)}$, using the SAS software was performed to identify the minimum number of observations ( $n 6$ ) required for the present pig experiment to reject the null hypothesis if this was false. Due to the missing washout period, all parameters, including ileal and faecal bacterial taxa and microbial metabolites, ileal digesta and faecal characteristics, passage rate, as well as ileal flow, hindgut disappearance and faecal excretion of nutrients, were analysed for potential carryover effects using a linear model and the MIXED procedure in SAS. This mixed model included the fixed effects of the sequence of feeding the diets, diet and replicate batch. The estimates showed that CON/TGS $v$. TGS/CON sequences had no significant impact on the data. Moreover, data of passage rate, ileal flow, hindgut disappearance and faecal excretion of nutrients, $\mathrm{pH}$, SCFA and lactate were analysed for normality using the Shapiro-Wilk test and outlier in SAS. ANOVA was performed using the MIXED procedure of SAS to compare the effects of TGS and CON diets with pig as the experimental unit. The model included the fixed effect of diet and the random effect of block. Degrees of freedom were approximated using the Kenward-Rogers method ( $\mathrm{ddfm}=\mathrm{kr}$ ). Pairwise comparisons among least square means were performed using the Tukey-Kramer test. Results are expressed as least square means with their standard error of the mean, and $P<0.05$ and $0.05<P \leq 0.10$ were defined as significance and trend, respectively.

\section{Results}

Based on pig's behaviour and appetite, all pigs remained clinically healthy throughout the experiment.
Table 2. Retention of digesta in the stomach and small intestine of pigs fed either the control (CON) diet ( $n 7)$ or transglycosylated starch (TGS) diet $(n 6)$ (Mean values with their standard errors)

\begin{tabular}{lcccc}
\hline Item & CON & TGS & SEM & $P$ \\
\hline $\begin{array}{l}\text { Liquid marker (Cr-EDTA) } \\
\text { Passage rate in the stomach }\end{array}$ & 0.0012 & 0.0018 & 0.0004 & 0.180 \\
(\%/h) & & & & \\
Passage rate in the small & 0.20 & 0.19 & 0.06 & 0.946 \\
intestine (\%/h) & & & & \\
Time delay (min) & 203 & 148 & 15.00 & $0.004^{\star *}$ \\
Peak flow (min) & 348 & 368 & 41.56 & 0.764 \\
GMRT (h) & 15.0 & 13.2 & 1.93 & 0.259 \\
SMRT (h) & 0.26 & 0.12 & 0.10 & 0.240 \\
TMRT (h) & 18.3 & 15.8 & 1.72 & 0.168 \\
Solid marker (Yb $\mathrm{O}_{3}$ ) & & & & \\
Passage rate in the stomach & 0.0010 & 0.0021 & 0.0015 & 0.102 \\
(\%/h) & & & & \\
Passage rate in the small & 0.11 & 0.75 & 0.15 & $0.017^{*}$ \\
intestine (\%/h) & & & & \\
Time delay (min) & 160 & 179 & 30.48 & 0.447 \\
Peak flow (min) & 287 & 355 & 35.19 & 0.291 \\
GMRT (h) & 16.3 & 11.3 & 1.64 & 0.064 \\
SMRT (h) & 0.22 & 0.03 & 0.05 & $0.039^{*}$ \\
TMRT (h) & 19.2 & 14.3 & 1.31 & 0.055 \\
\hline
\end{tabular}

GMRT, maximum gastric mean retention time; SMRT, maximum mean retention time in the small intestine; TMRT, maximum total mean retention time over the two compartments.

${ }^{\star} P<0.05,{ }^{\star \star} P<0.01$.

\section{Digesta retention time in the stomach and small intestine}

Both liquid (Cr-EDTA) and solid $\left(\mathrm{Yb}_{2} \mathrm{O}_{3}\right)$ phase markers were added to the morning meal on experimental day 16 to assess the transit through the stomach and small intestine. The average time delay until both liquid- and solid-phase markers appeared in ileal digesta was approximately 170 min after marker ingestion (Table 2), whereby the range of time delay was between 92 and 266 min for Cr-EDTA and between 85 and 299 min for $\mathrm{Yb}_{2} \mathrm{O}_{3}$ between both diets. Feeding of the TGS diet reduced time delay until the liquid fraction marker Cr-EDTA appeared in ileal digesta by 54 min compared with the CON diet $(P=0.004)$. With regard to the solid fraction, the fractional rate of passage of $\mathrm{Yb}_{2} \mathrm{O}_{3}$ in the small intestine (faster compartment) as a percentage per $\mathrm{h}$ was 6.9-fold greater with TGS but not different in the stomach compared with CON. Overall, the TGS diet caused a 5- and 0.2-h shorter GMRT $(P=0.064)$ and SMRT of digesta $(P=0.039)$, respectively, and subsequently tended $(P=0.055)$ to reduce TMRT compared with the CON diet.

\section{Intestinal flow and hindgut disappearance of proximate nutrients}

Pigs consumed the offered amount of feed, resulting in similar daily intake of DM, ash, starch, protein and energy between diets. Consumption of TGS increased the flow of DM, gross energy and starch $(P<0.001)$ and protein $(P=0.076)$ per $\mathrm{kg}$ of DM intake at the distal ileum by $2 \cdot 0-, 2 \cdot 0-$, 6.3- and 1.2-fold, respectively, compared with CON (Table 3). Although ileal flow of ash was similar between diets, the TGS diet increased the flow of $\mathrm{Ca}$ and $\mathrm{P}$ by 1.4- and 1.5-fold per $\mathrm{kg}$ of DM intake, respectively, compared with the CON diet. The disappearance of DM, starch and gross energy in the hindgut, in turn, 
Table 3. lleal flow, hindgut disappearance and faecal excretion of nutrients in pigs fed either the control (CON) diet $(n-7)$ or transglycosylated starch (TGS) diet $(n 6)$ (Mean values with their standard errors)

\begin{tabular}{|c|c|c|c|c|}
\hline Item & $\mathrm{CON}$ & TGS & SEM & $P$ \\
\hline \multicolumn{5}{|l|}{ Ileal flow (g/kg DM intake) } \\
\hline DM & 146 & 288 & $10 \cdot 50$ & $<0.001^{* \star *}$ \\
\hline Crude protein & 20 & 24 & 1.25 & 0.076 \\
\hline Crude ash & 25 & 27 & 1.12 & 0.390 \\
\hline Starch & 23 & 146 & 6.75 & $<0.001^{\star \star \star}$ \\
\hline $\mathrm{Ca}$ & 2.9 & 4.0 & 0.20 & $0.001^{\text {** }}$ \\
\hline $\mathrm{P}$ & 1.3 & $2 \cdot 0$ & 0.14 & $0.001^{* *}$ \\
\hline Gross energy (MJ/kg DM intake) & $2 \cdot 3$ & 4.6 & 0.15 & $<0.001^{\star \star \star}$ \\
\hline \multicolumn{5}{|c|}{ Hindgut disappearance (g/kg DM intake) } \\
\hline DM & 69 & 166 & $10 \cdot 85$ & $0.001^{\star * *}$ \\
\hline Crude protein & 11 & 6 & 1.79 & $0.047^{\star}$ \\
\hline Crude ash & 7 & 7 & 1.29 & 0.719 \\
\hline Starch & 23 & 123 & 8.48 & $<0.001^{\star \star *}$ \\
\hline $\mathrm{Ca}$ & 0.01 & 0.04 & 0.18 & 0.899 \\
\hline $\mathrm{P}$ & 0.02 & 0.01 & 0.12 & 0.961 \\
\hline Gross energy (MJ/kg DM intake) & 1.0 & 2.5 & 0.19 & $<0.001^{\star \star \star}$ \\
\hline \multicolumn{5}{|c|}{ Excreted in faeces (g/kg DM intake) } \\
\hline DM & 77 & 121 & 3.98 & $<0.001^{\star \star *}$ \\
\hline Crude protein & 9 & 18 & 0.84 & $<0.001^{\star \star *}$ \\
\hline Crude ash & 18 & 20 & 0.65 & 0.306 \\
\hline Starch & 0 & 22 & $2 \cdot 44$ & $<0.001^{\star \star \star}$ \\
\hline $\mathrm{Ca}$ & $2 \cdot 9$ & 3.9 & 0.14 & $0.004^{\star \star}$ \\
\hline $\mathrm{P}$ & 1.3 & $2 \cdot 0$ & 0.08 & $0.001^{\star *}$ \\
\hline Gross energy (MJ/kg DM intake) & 1.2 & $2 \cdot 1$ & 0.13 & $<0.001^{\star \star *}$ \\
\hline
\end{tabular}

${ }^{\star} P<0.05,{ }^{* \star} P<0.01,{ }^{* * *} P<0.001$.

was correspondingly higher in TGS- compared with CON-fed pigs, whereas TGS decreased hindgut disappearance of protein by 0.5 -fold $(P<0 \cdot 05)$. This resulted in a $1 \cdot 6-, 2 \cdot 0$ - and 1.75 -fold higher faecal excretion of DM, protein and gross energy in TGScompared with CON-fed pigs $(P<0 \cdot 001)$. Starch residuals could only be detected in faeces of TGS-fed pigs, which excreted $8 \%$ of the ingested starch, but not in faeces of CON-fed pigs $(P<0.001)$. Due to the unchanged large intestinal uptake, also 1.3- and 1.5-fold more $\mathrm{Ca}$ and $\mathrm{P}$ were excreted in faeces of TGS- compared with CON-fed pigs $(P<0 \cdot 01)$.

\section{Dry matter, $\mathrm{pH}$ and fermentation metabolites in ileal digesta and faeces}

Ileal digesta of TGS-fed pigs contained more DM compared with CON-fed pigs (online Supplementary Table S1), whereas in faeces the opposite relationship was found $(P<0.05$; online Supplementary Table S2). Moreover, the TGS diet decreased ileal and faecal $\mathrm{pH}$ by 0.4 and $2 \cdot 2$ log-units, respectively, compared with the CON diet (online Supplementary Tables S1 and S2). With respect to fermentation metabolites, the TGS diet enhanced total SCFA concentrations and SCFA output per kg of DM intake by two- to fivefold in ileal digesta and faeces $(P<0.05)$. This increase in total SCFA was mainly due to a largely enhanced acetate concentration at both intestinal sites with TGS compared with CON. Although the branched-chain fatty acids increased with the TGS diet in ileal digesta, the molar proportion of isobutyrate and isovalerate, similar to valerate, decreased with TGS compared with CON. Aside from the acetate concentration in faeces, faecal concentrations of butyrate and valerate

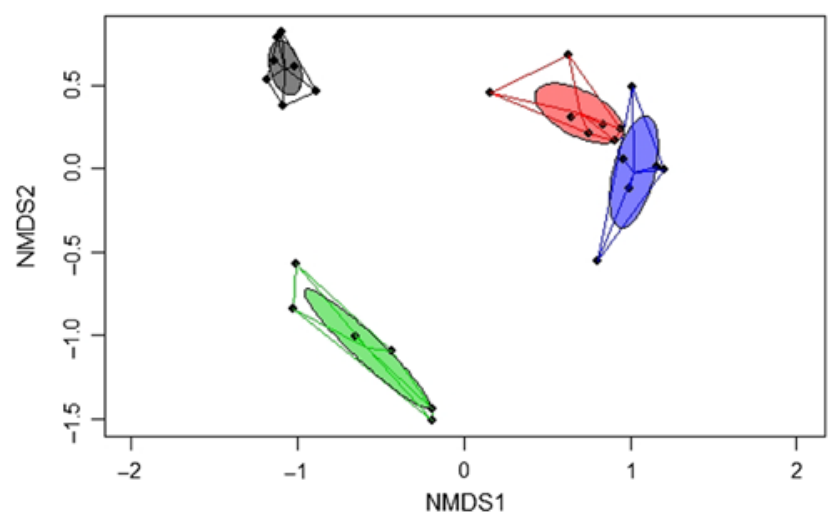

Fig. 1. Two-dimensional non-parametric multidimensional scaling (NMDS) ordination plots of ileal and faecal samples of pigs fed either the control (CON) or transglycosylated starch (TGS) diet ( $n 7$ CON diet; $n 6$ TGS diet). NMDS plots were generated using the Bray-Curtis distance metric between intestinal sites and diets. Each dot represents an individual sample; circles indicate the standard deviation. Red indicates ileal samples of CON-fed pigs; blue, ileal samples of TGS-fed pigs; grey, faecal samples of CON-fed pigs; and green, faecal samples of TGS-fed pigs.

increased by 5.4- and 3.9-fold, respectively, as well as to a smaller extent those of the branched-chain fatty acids in TGScompared with CON-fed pigs $(P<0 \cdot 05)$. When comparing faecal SCFA profiles as molar proportions of total SCFA, the TGS diet increased the proportions of acetate, butyrate and caproate but decreased the proportions of propionate and branchedchain fatty acids $(P<0 \cdot 05)$. Moreover, the TGS diet tended to enhance faecal total lactate, which was due to the tendency of a higher faecal s-lactate compared with the CON diet $(P<0 \cdot 10)$. In ileal digesta, the lesser abundant D-lactate was enhanced $(P<0.05)$ with TGS compared with CON.

\section{Composition of bacterial community}

A total of 3003839 high-quality sequences with a mean Phred score of 30-35 and a minimum of 25666 sequences per sample (mean, 75 096; mean length of stitched reads, 520 bp) were obtained for ileal and faecal samples when pigs were fed the TGS and CON diets. In total, 15 phyla were represented (data not shown). Read counts for the most abundant genera in ileal digesta and faeces can be found in online Supplementary Tables S3 and S4.

A significant separation of bacterial communities was detected in the Bray-Curtis-derived dissimilarity matrices (PERMANOVA) for ileal digesta and faeces $(P=0.002)$ and CON $v$. TGS diets $(P=0 \cdot 001)$. However, as indicated by the intestinal site $\times$ diet interaction $(P=0.001)$ in PERMANOVA, different clustering of bacterial communities in relation to pig's diet was mainly observed in faeces, which was visualised in the NMDS plots (Fig. 1). Likewise, ileal OTU richness and diversity were not affected by the dietary starch, whereas the diversity (Shannon and Simpson) of bacterial community in faeces of TGS-fed pigs was decreased $(P<0 \cdot 01)$ compared with CON-fed pigs (online Supplementary Table S5).

Of the predominant bacterial genera (Table 4), the TGS diet enriched the ileal community with Anaerovibrio, an unclassified 
Table 4. Selected bacterial genera (hit counts) in ileal digesta and faeces of pigs fed either the control diet $(n 7)$ or transglycosylated starch diet $(n 6)$ (Mean values with their standard errors)

\begin{tabular}{|c|c|c|c|c|c|}
\hline Item & Meant & $\log _{2}$-fold change & SE $\ddagger$ & $P$ & $q \S$ \\
\hline \multicolumn{6}{|l|}{ Ileal digesta } \\
\hline Anaerovibrio & 3430 & 8.024 & 1.321 & $<0.001^{\star * *}$ & $<0.001$ \\
\hline Unclassified Clostridiaceae & 1969 & -3.043 & 0.832 & $<0.001^{\star \star *}$ & 0.003 \\
\hline Unclassified Veillonellaceae & 677 & 6.852 & $1 \cdot 110$ & $<0.001^{\star \star *}$ & $<0.001$ \\
\hline Megasphaera & 531 & $5 \cdot 240$ & 1.153 & $<0.001^{\star \star *}$ & $<0.001$ \\
\hline Klebsiella & 325 & 4.165 & 1.464 & $0.004^{\star \star}$ & 0.021 \\
\hline Ruminococcus & 246 & $-2 \cdot 170$ & 0.601 & $<0.001^{\star \star \star}$ & 0.003 \\
\hline Unclassified Clostridiales & 199 & $-2 \cdot 375$ & 0.657 & $<0.001^{\star \star \star}$ & 0.003 \\
\hline Unclassified Ruminococcaceae & 148 & $-2 \cdot 359$ & 0.655 & $<0.001^{\star \star \star}$ & 0.003 \\
\hline Unclassified Christensenellaceae & 127 & -1.964 & 0.581 & $0.001^{\star \star}$ & 0.004 \\
\hline Selenomonas & 123 & $1 \cdot 702$ & 0.743 & $0.022^{*}$ & 0.077 \\
\hline Lactobacillus & 122 & $3 \cdot 220$ & 0.857 & $<0.001^{\star * *}$ & 0.003 \\
\hline Mitsuokella & 91 & 5.082 & 0.968 & $<0.001^{\star \star *}$ & $<0.001$ \\
\hline Sutterella & 80 & $-2 \cdot 799$ & 1.262 & $0.027^{\star}$ & 0.083 \\
\hline Unclassified Coriobacteriaceae & 64 & 3.714 & 0.881 & $<0.001^{\star * *}$ & 0.001 \\
\hline Succinivibrio & 32 & -0.440 & 0.689 & $<0.001^{\star \star *}$ & $<0.001$ \\
\hline Unclassified Desulfovibrionaceae & 21 & -5.006 & 1.020 & $<0.001^{\star \star \star}$ & $<0.001$ \\
\hline Unclassified Bacteroidales & 16 & $-4 \cdot 644$ & 1.371 & $0.001^{\star *}$ & 0.004 \\
\hline Unclassified Alcaligenaceae & 14 & -3.442 & 1.518 & $0.023^{*}$ & 0.079 \\
\hline rc4.4 & 11 & $-5 \cdot 552$ & 1.507 & $<0.001^{\star \star \star}$ & 0.003 \\
\hline Pyramidobacter & 10 & $-5 \cdot 058$ & 1.843 & $0.006^{\star \star}$ & 0.027 \\
\hline Pseudomonas & 10 & -4.443 & 1.361 & $0.001^{\star *}$ & 0.006 \\
\hline \multicolumn{6}{|l|}{ Faeces } \\
\hline Unclassified Christensenellaceae & 13296 & $-3 \cdot 263$ & 0.824 & $<0.001^{\star \star \star}$ & $<0.001$ \\
\hline Unclassified Ruminococcaceae & 8598 & $-2 \cdot 562$ & 0.854 & $0.003^{\star \star}$ & 0.007 \\
\hline Unclassified Clostridiales & 6788 & $-3 \cdot 375$ & 0.652 & $<0.001^{\star \star *}$ & $<0.001$ \\
\hline Unclassified Coriobacteriaceae & 4778 & $7 \cdot 192$ & 1.025 & $<0.001^{\star \star \star}$ & $<0.001$ \\
\hline Ruminococcus & 4049 & $1 \cdot 281$ & 0.577 & $0.026^{*}$ & 0.053 \\
\hline Megasphaera & 3582 & 8.085 & 0.696 & $<0.001^{\star \star \star}$ & $<0.001$ \\
\hline Unclassified Enterobacteriaceae & 3395 & $-2 \cdot 409$ & 0.587 & $<0.001^{\star \star *}$ & $<0.001$ \\
\hline Desulfovibrio & 534 & 1.504 & 0.576 & $0.009^{\star *}$ & 0.023 \\
\hline Unclassified Veillonellaceae & 533 & 4.257 & 1.025 & $<0.001^{\star \star \star}$ & $<0.001$ \\
\hline Unclassified $p-2534-18 B 5$ & 487 & $-10 \cdot 542$ & $1 \cdot 191$ & $<0.001^{\star \star *}$ & $<0.001$ \\
\hline Unclassified Clostridiaceae & 276 & $-5 \cdot 322$ & 0.738 & $<0.001^{\star \star *}$ & $<0.001$ \\
\hline Oscillospira & 236 & -3.429 & 0.841 & $<0.001^{\star \star *}$ & $<0.001$ \\
\hline Unclassified RF39 & 228 & $-4 \cdot 344$ & 1.339 & $0.001^{\star *}$ & 0.003 \\
\hline Collinsella & 212 & 3.554 & 0.799 & $<0.001^{\star \star \star}$ & $<0.001$ \\
\hline Mitsuokella & 204 & 6.733 & $1 \cdot 120$ & $<0.001^{\star \star *}$ & $<0.001$ \\
\hline RFN20 & 199 & 2.935 & 0.688 & $<0.001^{\star \star *}$ & $<0.001$ \\
\hline Succinivibrio & 199 & -6.083 & $1 \cdot 140$ & $<0.001^{\star \star *}$ & $<0.001$ \\
\hline Sutterella & 87 & 1.389 & 0.647 & $0.032^{*}$ & 0.060 \\
\hline rc4.4 & 61 & -7.928 & 1.061 & $<0.001^{\star \star \star}$ & $<0.001$ \\
\hline Catenibacterium & 41 & 7.727 & 1.245 & $<0.001^{\star \star *}$ & $<0.001$ \\
\hline Dorea & 39 & -7.995 & 0.987 & $<0.001^{\star * *}$ & $<0.001$ \\
\hline Blautia & 32 & 1.753 & 0.782 & $0.025^{\star}$ & 0.053 \\
\hline Unclassified GMD14H09 & 31 & $-8 \cdot 355$ & $2 \cdot 086$ & $<0.001^{\star \star \star}$ & $<0.001$ \\
\hline Unclassified Pirellulaceae & 27 & -4.439 & $1 \cdot 287$ & $0.001^{\star \star \star}$ & 0.002 \\
\hline Unclassified $R 4-45 B$ & 17 & -8.017 & 1.392 & $<0.001^{\star \star \star}$ & $<0.001$ \\
\hline Unclassified Victivallaceae & 16 & $-3 \cdot 494$ & 0.969 & $<0.001^{\star \star \star}$ & 0.001 \\
\hline Dehalobacterium & 16 & $-7 \cdot 892$ & $1 \cdot 169$ & $<0.001^{\star \star \star}$ & $<0.001$ \\
\hline Unclassified Mogibacteriaceae & 13 & $-3 \cdot 817$ & 0.881 & $<0.001^{\star \star \star}$ & $<0.001$ \\
\hline Coprococcus & 10 & -1.895 & 0.881 & $0.031^{*}$ & 0.060 \\
\hline
\end{tabular}

${ }^{\star} P<0.05,{ }^{* \star} P<0.01,{ }^{* \star *} P<0.001$.

$\dagger$ Normalised reads (hit counts).

$\ddagger$ Standard error of the $\log _{2}$-fold change.

$\S$ False discovery rate (Benjamini-Hochberg)-corrected $P$ value.

Veillonellaceae genus, Megasphaera, Klebsiella, Lactobacillus, Mitsuokella, an unclassified Coriobacteriaceae genus $(P<0 \cdot 01$; $q<0.05)$ and Selenomonas $(P<0.05 ; q<0.1)$ compared with the CON diet. By contrast, TGS-fed pigs comprised less reads of an unclassified Clostridiaceae genus, Ruminococcus and unclassified genera belonging to Clostridiales, Ruminococcaceae and Christensenellaceae $(P<0.01 ; q<0.05)$ as well as of Sutterella $(P<0.05 ; q<0 \cdot 1)$ in their ileal digesta compared with CON-fed pigs $(P<0 \cdot 05 ; q<0 \cdot 1)$. Due to the divergence of the faecal bacterial community from ileal community (Fig. 1), the effects of TGS on faecal bacterial community were partly different between the two sites. In faeces (Table 4), the TGS diet enriched $(P<0.001 ; q<0.05)$ the community especially with reads from unclassified Coriobacteriaceae $\left(+7 \cdot 19 \quad \log _{2}\right.$-fold changes), Megasphaera (+8.09 $\log _{2}$-fold changes) and Mitsuokella (+6.73 $\log _{2}$-fold changes) and, to a lesser extent, 
(a)

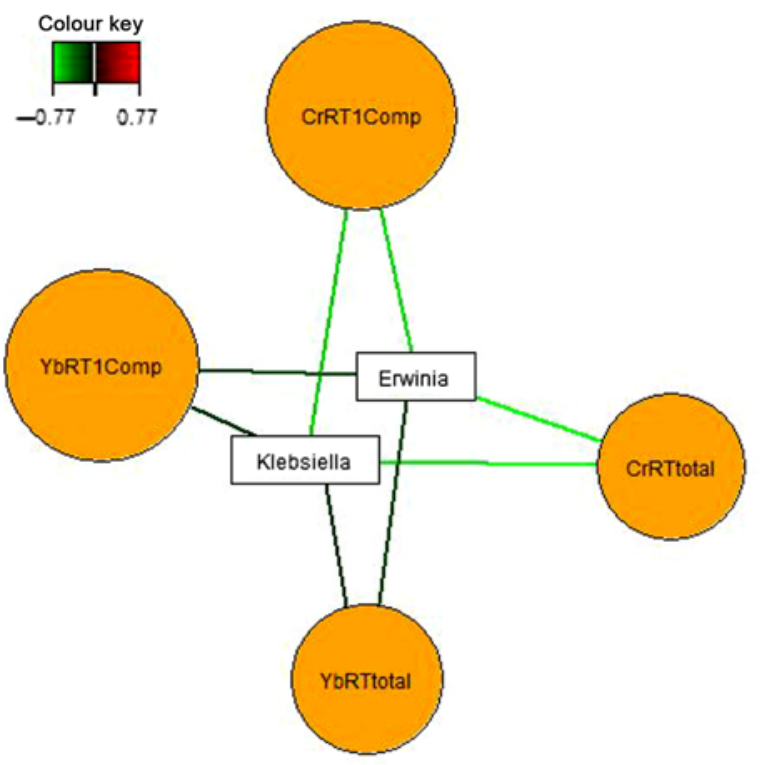

(c)

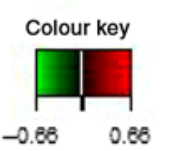

(b)

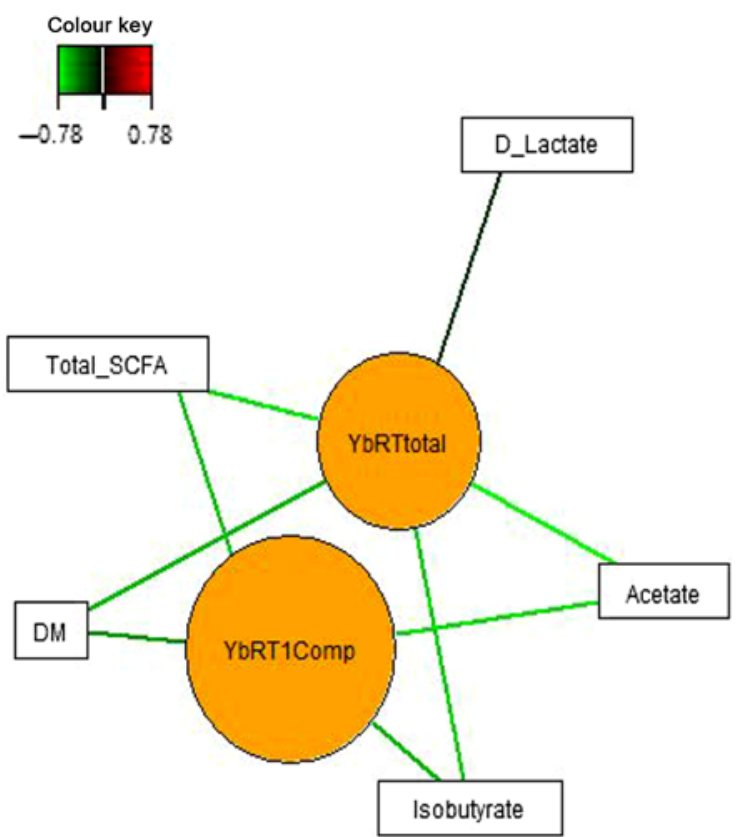

(d)

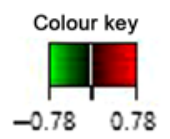

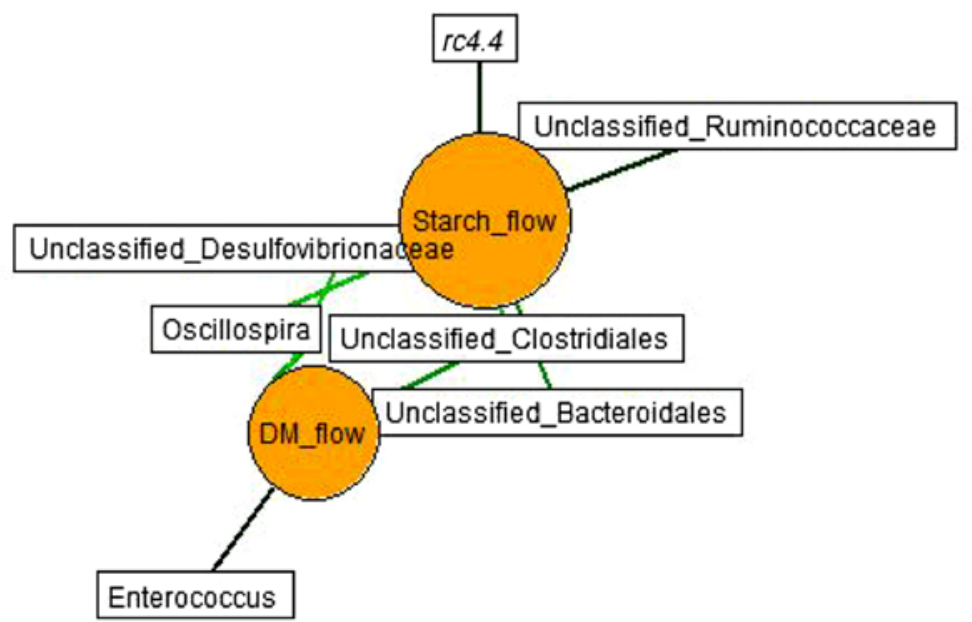

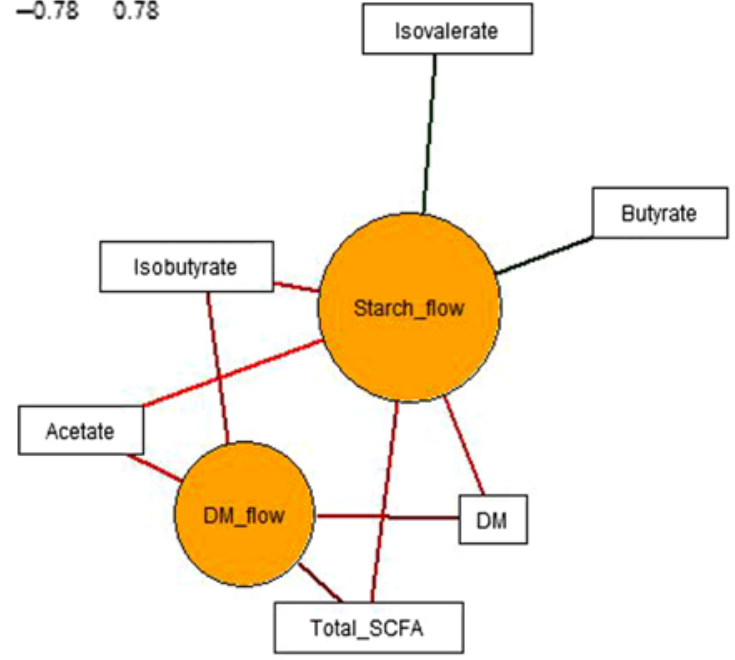

Fig. 2. Dependencies of bacterial genera and fermentation metabolites from digesta retention time and nutrient flow in ileal digesta (a-d). Covariations between the retention time in the stomach and small intestine, ileal nutrient flow, relative abundances of bacterial genera (relative abundance $>0.01 \%$ of all reads) and fermentation metabolites including digesta $\mathrm{pH}$ and DM content were established separately using sparse partial least squares regression and relevance networking. The networks are displayed graphically as nodes (parameters) and edges (biological relationship between nodes). The edge colour intensity indicates the level of association: red = positive, green = negative. Only the strongest pairwise associations were projected $(I H>0.6)$. CrRT1Comp, liquid marker fractional passage rate for compartment 1 (stomach); CrRTtotal, liquid marker fractional passage rate for both compartments; YbRT1Comp, solid marker fractional passage rate for compartment 1 (stomach); YbRTtotal, solid marker fractional passage rate for both compartments.

with Ruminococcus, Desulfovibrio, an unclassified Veillonellaceae genus and Collinsella $(P<0.05 ; q<0.1)$. Dominant bacterial genera that decreased with the TGS diet were, like in ileal digesta, unclassified genera belonging to Clostridiales, Ruminococcaceae, Christensenellaceae and Enterobacteriaceae $(P<0.01 ; q<0.05)$.
Relevance networks and sparse partial least squaresdiscriminant analysis data integration between bacterial genera, SCFA and nutrient flow

Sparse partial least squares regression and relevance networking were used to identify bacterial genera and SCFA that were most 
(a)

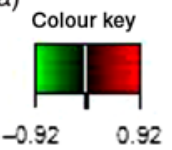

(b)

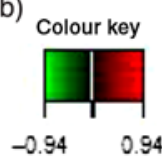

Fig. 3. Dependencies of bacterial genera (a) and fermentation metabolites (b) from nutrient flow (excretion) in faeces. Covariations between faecal nutrient flow (excretion), relative abundances of bacterial genera (relative abundance $>0.01 \%$ of all reads) and fermentation metabolites including faecal pH and $\mathrm{DM}$ content were established separately using sparse partial least squares regression and relevance networking. The networks are displayed graphically as nodes (parameters) and edges (biological relationship between nodes). The edge colour intensity indicates the level of association: red = positive, green = negative. Only the strongest pairwise associations were projected $(|r|>0 \cdot 8)$. CP, crude protein.

influenced by ileal retention time and ileal (Fig. 2) and faecal nutrient flow (Fig. 3). The exact covariation scores for these associations can be found in online Supplementary Tables S6 and S7. Networks showed that Klebsiella and Erwinia were negatively associated with the retention time of the solid- and liquid-phase markers in digesta, with the retention of digesta in compartment 1 (stomach) being the most influential ( $|r|>0 \cdot 6$; Fig. 2(a)). The retention of the solid-phase marker, again especially the retention in the stomach, also negatively correlated with ileal concentrations of total SCFA, acetate and isobutyrate and ileal DM content, whereas D-lactate was negatively associated with the retention of the solid phase in both compartments $(|r|>0 \cdot 6$; Fig. 2(b)). Ileal abundances of five genera (Oscillospira, Enterococcus and unclassified genera within Desulfovibrionaceae, Clostridiales and Bacteroidales) were negatively associated with ileal DM flow, which together with unclassified Ruminococcaceae genus and $r c 4.4$ (except Enterococcus) also negatively correlated with ileal starch flow $(|r|>0 \cdot 6$; Fig. 2(c)). Positive dependencies with ileal DM and starch flow existed for total SCFA, acetate, isobutyrate and ileal DM content, whereas negative relationships existed between ileal starch flow and ileal butyrate and isovalerate concentrations $(|r|>0 \cdot 6$; Fig. 2(d)). In faecal samples, Megasphaera, Dialister and Catenibacterium were positively, and Oscillospira and an unclassified Ruminococcaceae genus negatively, associated with faecal DM, starch and protein flow (excretion) $(|r|>0 \cdot 6$; Fig. 3(a)). Oscillospira was additionally negatively linked with faecal $P$ excretion, and an unclassified Victivallaceae genus with faecal ash flow (excretion) $(|r|>0 \cdot 6)$. Total SCFA, acetate, butyrate and caproate were positively associated with DM, starch and protein flow (excretion) in faeces, whereby total SCFA and acetate were also positively associated with $\mathrm{Ca}$ and $\mathrm{P}$ excretion, and butyrate with faecal $\mathrm{P}$ excretion $(|r|>0 \cdot 6$; Fig. 3(b)). Valerate was mainly positively associated with faecal protein excretion $(|r|>0 \cdot 6)$. Oppositely, faecal pH was negatively associated with DM, starch, protein and $\mathrm{P}$ excretion in faeces $(|r|>0 \cdot 6)$.

In order to obtain a better understanding of the dependencies among nutrient flow in the digesta and bacterial activity, we integrated the various datasets using the 'tune' function in sPLS-DA to model relationships between the different data that achieved the best predictive performance ${ }^{(29)}$. Dietary starch-associated separation of the most discriminant genera (block: genus), fermentation metabolites (block: SCFA) and nutrient flow (block: $\mathrm{NF}$ ) were visualised in sample plots per dataset; loading plots and relationships among the identified, most discriminant features were illustrated in circos plots for component 1 . Sample plots indicated that, despite a clear separation between diets for the nutrient flow, diet-related clusters for genera and SCFA still overlapped in ileal digesta and only were clearly separated in faeces (online Supplementary Fig. S2). Loading plots showed that unclassified genera of Synergistaceae and Desulfovibrionaceae, acetate and starch flow were the most influential features in ileal digesta, whereas Oscillospira, 
(a)

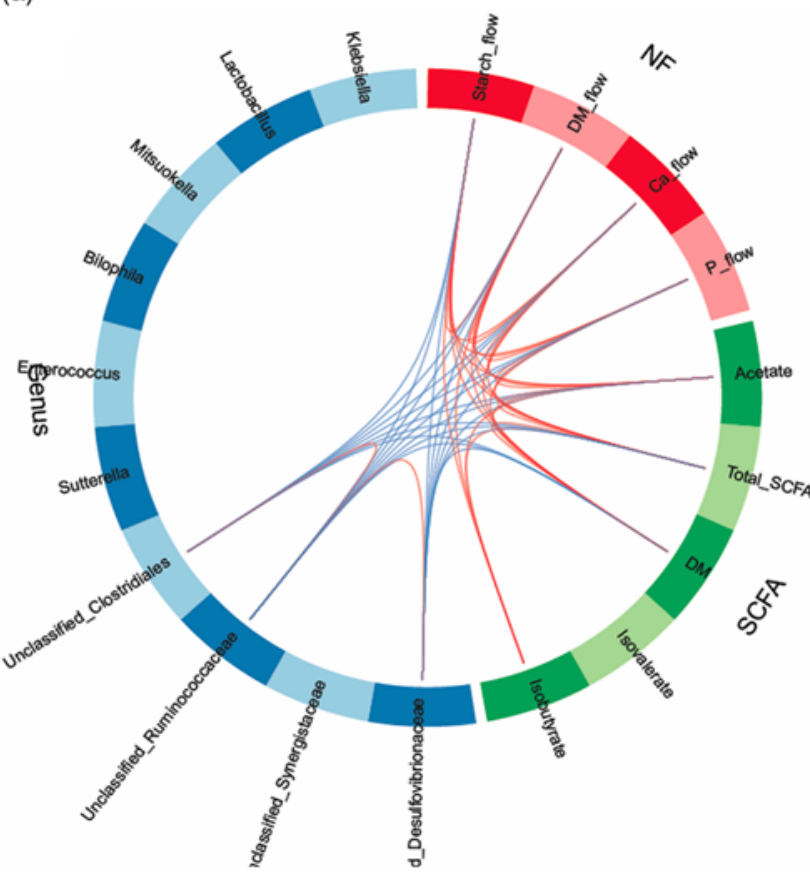

(b)

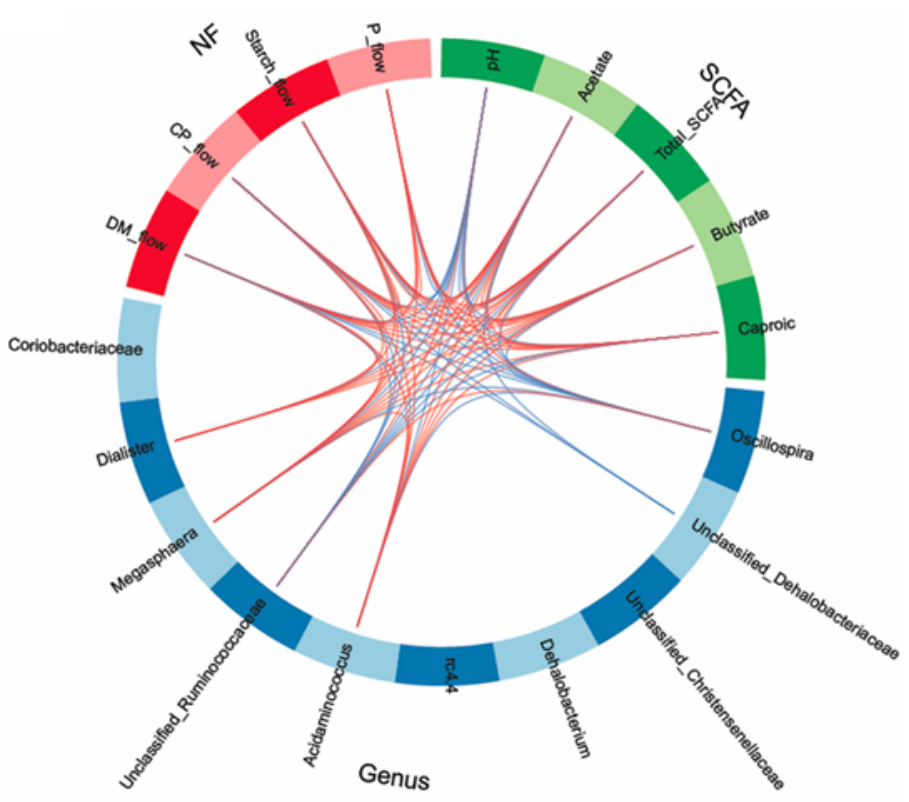

Fig. 4. Circos plots of horizontal sparse partial least squares-discriminant analysis displaying correlations between the identified best discriminant genera ( $n$ 10), SCFA $(n 5)$ and nutrient flow (NF) $(n 4)$ for component 1 in ileal digesta (a) and faeces (b). Positive and negative correlations ( $|r|>0.6$ for ileum and $|r|>0.8$ for faeces) are displayed by red and blue links, respectively. Relative abundance of bacterial genera $>0.01 \%$ of all reads. CP, crude protein.

Megasphaera, $\mathrm{pH}$ and DM excretion were the most discriminant variables in faeces for component 1 (online Supplementary Fig. S3). More relationships between the most influential parameters were detected in faeces than in ileal digesta, supporting the relationships between nutrient flow with SCFA concentrations and the best discriminant genera (Fig. 4).

\section{Discussion}

Dietary fibres, including RS, often alter the nutrient flow and bulk of intestinal digesta, thereby changing the substrate availability to the microbiota along the gastrointestinal tract ${ }^{(13,33)}$. By greatly increasing ileal starch flow and substrate availability for fermentation in the hindgut, the present data demonstrated low intestinal digestibility and the RS properties of TGS. Taxonomic shifts related to TGS consumption occurred mostly within the Firmicutes and Proteobacteria phyla at both intestinal sites. We could associate several bacterial genera with ileal and faecal nutrient flow, showing that the intestinal bacteria adapt rapidly to the dietary starch source. Our results further emphasise the importance of the retention time of digesta for ileal bacterial composition and metabolic activity. In contrast to our hypothesis, TGS did not slow down transit in the stomach and small intestine but accelerated passage in the upper digestive tract, leaving less time for bacterial action on TGS. Relevance networking suggested that especially Proteobacteria genera, as the predominant Klebsiella, benefitted from the faster transit of digesta in the stomach. A shorter retention in the upper digestive tract may explain the small Bray-Curtis-derived distances between the bacterial communities in ileal digesta compared with faeces between
CON- and TGS-fed pigs. Nevertheless, the lower $\mathrm{pH}$ and elevated SCFA concentrations in ileal digesta provide evidence for the microbial use of TGS in the upper digestive tract. Overall, when interpreting the present results, it should be kept in mind that, despite randomising the pigs to dietary treatments in both replicate batches and the absence of carryover effects, it cannot be completely ruled out that a certain residual effect may have existed, thereby biasing the direct treatment effect.

TGS mainly reduced the retention of solid digesta with little impact on the liquid fraction in the upper digestive tract. Due to the sevenfold faster transit of the solid fraction, it is reasonable to assume that, besides starch, other dietary components may have been less completely digested and absorbed in the upper digestive tract of TGS-fed pigs, as well. Results for ileal nutrient flow showed that mainly the digestion of starch, Ca and $\mathrm{P}$ was impaired by TGS, whereas the reduced retention of digesta in the stomach and small intestine did not alter protein digestion. The enhanced ileal flow of starch increased the DM content of digesta as indicated by the present correlations. Although some residual starch may have originated from the waxy maize starch, the digestibility coefficients led us to assume that most starch entering the large intestine were TGS residuals. We did not measure the viscosity of ileal digesta and can only speculate whether increased digesta viscosity contributed to a higher ileal DM content with TGS. Oppositely, the DM content of faeces was lower, indicating certain bulking properties of TGS as, in total, more DM was excreted by TGS- compared with CON-fed pigs. Since fermentation acids have osmotic properties, the greater water content in faeces of TGS-fed pigs may have been partly caused by their threefold higher faecal SCFA and lactate 
concentrations. Faecal bulking properties may be attractive for using TGS as a functional food in human nutrition ${ }^{(2,3)}$. For this, its efficacy at lower dosages as a supplement or food ingredient needs to be established first.

According to ileal networks, the retention of digesta in the stomach was more important for bacterial action than the retention in the small intestine. The networks indicated that Klebsiella and less abundant Erwinia benefitted from the altered conditions (i.e. fermentable substrate and time for fermentation) in the upper digestive tract of TGS-fed pigs. Klebsiella produces starch-hydrolysing and debranching pullulanase enzymes and can utilise starch as the sole carbon and energy source ${ }^{(34)}$. This may have given this genus a growth advantage. Nevertheless, TGS had a stronger effect on faecal bacterial community than on ileal community as indicated by similar OTU richness and diversity in the ileum but reduced evenness of the bacterial community in faeces. The drastic shifts in the predominant bacterial genera in ileal digesta and faeces clearly emphasised that different enzymatic capabilities were needed for the degradation of TGS compared with the waxy maize starch. A recent functional analysis by our group using whole-genome shotgun metagenomics indicated steps in the breakdown of TGS by the action of $\alpha$ - and $\beta$-galactosidases, which replaced genes coding for enzymes within the starch and sucrose metabolism $^{(35)}$.

Although similar TGS effects were identifiable for ileal digesta and faeces, intestinal site-specific shifts were clearly detectable, which can be related to the retention time and changes in substrate flow with continuous digestion and fermentation along the gastrointestinal tract. The present results for TGS-related shifts in ileal and faecal bacterial communities were per se comparable to our previous study using a slaughter pig model ${ }^{(9)}$. Nevertheless, taxonomic differences between both studies existed, which may be related to the pig model and inter-individual microbiome composition among pigs. Aside from Klebsiella, mainly saccharolytic and amylolytic genera, such as Anaerovibrio, Selenomonas, Lactobacillus and Coriobacteriaceae, benefitted from TGS in ileal digesta. These taxa produce lactate, acetate and succinate as fermentation end-products ${ }^{(36-38)}$, which would correspond to the present results for fermentation acids in the ileum. A TGS-related increase in Klebsiella and Coriobacteriaceae, however, needs to be critically monitored in human studies as both taxa may act pro-inflammatory and have been linked to inflammatory bowel disease and other autoimmune diseases $^{(24,39)}$. A TGS-related rise in the Actinobacteria genus Collinsella, identified as another discriminant genus in ileal digesta and probably able to degrade glycosidic bonds in TGS, in turn, may be beneficial for intestinal health through its production of SCFA and hippurate ${ }^{(40)}$. sPLS-DA also emphasised the Veillonellaceae genus Dialister as important taxa for TGS degradation in faeces. Previously, Dialister has been related to the anti-inflammatory effect of whole-grain diets as increased numbers of this genus have been associated with lower IL-6 levels in the plasma of humans ${ }^{(41)}$. Similarly, we recently reported the anti-inflammatory properties of TGS and could link an enhanced Dialister abundance with lower toll-like receptor- 4 expression in the colon of TGS-fed pigs ${ }^{(16)}$.
Moreover, due to progressing digestion and fermentation, it can be assumed that various stages of degradation products from TGS reached the distal colon, thereby stimulating the activity of other amylolytic and saccharolytic species that were otherwise not able to use TGS. This may explain the opposite findings for a decline in Ruminococcus in ileal digesta and their enrichment in faeces of TGS-fed pigs.

Aside from starch and TGS degradation products, complex interactions among the bacteria such as primary metabolite cross-feeding, for example, of lactate and succinate ${ }^{(42)}$, may explain the increased hit counts for Veillonellaceae, Mitsuokella and Megasphaera in ileal digesta and faeces. Their increase likely contributed to the elevated faecal concentrations of acetate, butyrate, valerate and caproate ${ }^{(43)}$ with TGS feeding, thereby compensating the lower contribution of taxa that thrived less well on TGS to the ileal and faecal SCFA concentrations, such as genera within Ruminococcaceae and Clostridiaceae, according to the present correlations. In faeces, butyrate-producing Oscillospira within the Ruminococcaceae family ${ }^{(44)}$ was the most discriminating genus, but decreased by TGS. This is interesting as this genus has been reported to increase during fasting and on lower-starch diets by feeding mostly on host glycans ${ }^{(44)}$. Therefore, an indirect effect via the modification of the host glycan expression may explain the TGS-related decline in Oscillospira abundance, which would be in line with our previous observation for a $61 \%$ lower mucin 2 expression at the caecal mucosa in TGS- compared to CON-fed pig $^{(16)}$. A similar dependency may explain the reduced abundance of Christensenellaceae in ileal digesta and faeces of TGS- compared with CON-fed pigs. As a consequence of increased fermentation of residual starch, TGS lowered ileal and faecal $\mathrm{pH}$, which may have also modified bacterial abundances and their metabolic activity ${ }^{(42)}$.

Although acetate was the best discriminant fermentation acid at both intestinal sites, a clear relationship with the most discriminant bacteria in ileal digesta was missing. From the intestinal networks, it can be perceived that Megasphaera is a major taxa contributing to faecal butyrate and caproate levels, whereas Collinsella and the Lachnospiraceae genus Blautia appear to be important contributors to faecal acetate. While butyrate constitutes the primary energy source for enterocytes, acetate is transported via the portal vein to the liver where it can be directly used or is transported to adipocytes for de novo lipogenesis ${ }^{(45,46)}$. Before being absorbed, SCFA (e.g. acetate, propionate and butyrate) activate $\mathrm{G}$-protein receptors, which regulate immune and gene transcription pathways ${ }^{(4)}$. Consequently, the attenuation of intestinal inflammation by SCFA may be one mechanism how the consumption of TGS may benefit host physiology. Another advantage of TGS may be its slow fermentation along the hindgut as indicated by the residual starch excreted in faeces, nourishing the epithelium in the distal colon-rectum region with straight-chain fatty acids ${ }^{(4)}$. Concurrently, the negative impact of protein fermentation (e.g. via ammonia, amines, phenols and sulphides) on the functioning and integrity of colonic epithelium $^{(47,48)}$ may have been reduced in TGS- $v$. CON-fed pigs as indicated by the lower molar proportions of branched-chain fatty acids and hindgut disappearance of protein with the TGS diet. 
Several scenarios may explain the increased flow of Ca and $\mathrm{P}$ into the large intestine with the TGS diet. It is probable that TGS has greater mineral-chelating abilities than the waxy maize starch used as control, or that a higher digesta viscosity with TGS reduced the absorption of $\mathrm{Ca}$ and $\mathrm{P}$ until the ileum. A shorter retention in the stomach of TGS-fed pigs together with a high buffering capacity of the diet (casein and minerals) may have impaired a proper dissociation into $\mathrm{Ca}^{2+}$ and $\mathrm{PO}_{4}^{3-}$ ions in the gastric content, thereby lowering their absorption in the small intestine. It may be also speculated whether bacterial incorporation $^{(14)}$ contributed to a greater flow of $\mathrm{Ca}$ and $\mathrm{P}$ in ileal digesta and faeces of TGS-fed pigs as these minerals are essential co-factors and constituents in bacterial cells. In line with that, relevance networks indicated the importance of faecal Ca for acetate and that of $\mathrm{P}$ for acetate and butyrate, possibly indicating bacterial mineral needs for fermentation ${ }^{(49)}$. If similar effects on intestinal mineral absorption are observed with lower and more reasonable dietary inclusion levels of TGS (e.g. $5 \%$ ), this may require extra supplementation of diets with $\mathrm{Ca}$ and $\mathrm{P}$. In this line, it would be also valuable to evaluate whether TGS impacts the dietary availability of other macro- and trace elements.

In conclusion, the present results showed that the consumption of TGS caused distinct bacterial composition and metabolite profiles in ileal digesta and faeces, which were modulated by the type of starch, intestinal starch flow and faster transit of digesta in the stomach and small intestine. Relevance networks indicated that Klebsiella in ileal digesta may have benefitted from the reduced retention of digesta in the stomach. Data integration analysis further suggested dependencies between faecal nutrient (starch) availability, promotion of Coriobacteriaceae, Collinsella, Blautia and Megasphaera and increase in SCFA, especially acetate and butyrate. Taken together, the consumption of TGS showed certain benefits for the host health, such as increased faecal bulk and carbohydrate fermentation in the distal hindgut. However, its stimulation of certain bacterial taxa that were previously linked to inflammatory diseases (e.g. Klebsiella and Coriobactericeae) needs to be meticulously tested using lower dietary inclusion levels in animal models and human volunteers.

\section{Supplementary material}

To view supplementary material for this article, please visit https://doi.org/10.1017/S0007114519000849

\section{Acknowledgements}

We thank Arife Sener, Suchitra Sharma, Anita Dockner and Melanie Wild (Institute of Animal Nutrition and Functional Plant Compounds) for their technical assistance. Christian Knecht and Elena Sassu (University Clinic for Swine) are thanked for their assistance during surgery. Timea Molnar-Wegerth is thanked for assistance with starch provision and analysis (Agrana Research \& Innovation Center GmbH). We further thank Agromed Austria GmbH for providing the cellulose (FibroCell ${ }^{\circledR}$ M1) to be incorporated into the diets.
This work was supported by the Austrian Research Promotion Agency (FFG) (BRIDGE project no. 836447 'Healthy Carbohydrates').

B. U. M.-Z., D. G. and Q. Z. designed the research; B. U. M.-Z. and M. A. N. conducted the research; W. K. performed passage rate marker analysis; B. U. M.-Z. and M. A. N. performed bioinformatics with QIIME; B. U. M.-Z. performed data analysis in R and SAS; B. U. M.-Z. wrote the paper; and B. U. M.-Z. had primary responsibility for the final content. All authors read and approved the final manuscript.

Agrana Research \& Innovation Center GmbH provided support in the form of salary to author D.G., but did not have any additional role in the study design, data collection and analysis, decision to publish or preparation of the manuscript.

\section{References}

1. Bach Knudsen KE, Lærke HN, Hedemann MS, et al. (2018) Impact of diet-modulated butyrate production on intestinal barrier function and inflammation. Nutrients 10, E1499.

2. Topping DL \& Clifton PM (2001) Short-chain fatty acids and human colonic function: roles of resistant starch and nonstarch polysaccharides. Physiol Rev 81, 1031-1064.

3. Birt DF, Boylston T, Hendrich S, et al. (2013) Resistant starch: promise for improving human health. Adv Nutr 4, 587-601.

4. McKenzie C, Tan J, Macia L, et al. (2017) The nutrition-gut microbiome-physiology axis and allergic diseases. Immunol Rev 278, 277-295.

5. Wilfart A, Montagne L, Simmins H, et al. (2007) Digesta transit in different segments of the gastrointestinal tract of pigs as affected by insoluble fibre supplied by wheat bran. BrJ Nutr 98, 54-62.

6. Souza da Silva C, Haenen D, Koopmans SJ, et al. (2014) Effects of resistant starch on behaviour, satiety-related hormones and metabolites in growing pigs. Animal 8, 1402-1411.

7. Ingerslev AK, Mutt SJ, Lærke HN, et al. (2017) Postprandial PYY increase by resistant starch supplementation is independent of net portal appearance of short-chain fatty acids in pigs. PLOS ONE 12, e0185927.

8. Newman MA, Zebeli Q, Eberspächer E, et al. (2017) Transglycosylated starch improves insulin response and alters lipid and amino acid metabolome in a growing pig model. Nutrients 9, 291.

9. Newman MA, Petri RM, Grüll D, et al. (2018) Transglycosylated starch modulates the gut microbiome and expression of genes related to lipid synthesis in liver and adipose tissue of pigs. Front Microbiol 9, 224.

10. Guilloteau P, Zabielski R, Hammon HM, et al. (2010) Nutritional programming of gastrointestinal tract development: is the pig a good model for man? Nutr Res Rev 23, 4-22.

11. Nielsen KL, Hartvigsen ML, Hedemann MS, et al. (2014) Similar metabolic responses in pigs and humans to breads with different contents and compositions of dietary fibers: a metabolomics study. Am J Clin Nutr 99, 941-949.

12. Patterson JK, Lei XG \& Miller DD (2008) The pig as an experimental model for elucidating the mechanisms governing dietary influence on mineral absorption. Exp Biol Med (Maywood) 233, 651-664.

13. Newman MA, Zebeli Q, Velde K, et al. (2016) Enzymatically modified starch favorably modulated intestinal transit time and hindgut fermentation in growing pigs. PLOS ONE 11, e0167784.

14. Metzler BU, Mosenthin R, Baumgärtel T, et al. (2008) The effect of dietary phosphorus and calcium level, phytase 
supplementation, and ileal infusion of pectin on the chemical composition and carbohydrase activity of fecal bacteria and the level of microbial metabolites in the gastrointestinal tract of pigs. J Anim Sci 86, 1544-1555.

15. National Research Council (2012) Nutrient Requirements of Swine, 11th ed. Washington, DC: National Academies Press.

16. Metzler-Zebeli BU, Newman MA, Grüll D, et al. (2018) Consumption of transglycosylated starch down-regulates expression of mucosal innate immune response genes in the large intestine using a pig model. Br J Nutr 119, 1366-1377.

17. AOAC (2006) Official Methods of Analysis, 18th ed. Arlington, VA: Association of Official Analytical Chemists

18. Naumann C \& Basler R (2012) Die Chemische Untersuchung von Futtermitteln (The Chemical Investigation of Feed), 3rd ed. Darmstadt, Germany: VDLUFA Verlag.

19. Khol-Parisini A, Humer E, Sizmaz Ö, et al. (2015) Ruminal disappearance of phosphorus and starch, reticuloruminal $\mathrm{pH}$ and total tract nutrient digestibility in dairy cows fed diets differing in grain processing. Anim Feed Sci Technol 210, 74-85.

20. Zebeli Q, Tafaj M, Weber I, et al. (2007) Effects of varying dietary forage particle size in two concentrate levels on chewing activity, ruminal mat characteristics, and passage in dairy cows. J Dairy Sci 90, 1929-1942.

21. Metzler-Zebeli BU, Lawlor PG, Magowan E, et al. (2016) Effect of freezing conditions on fecal bacterial composition in pigs. Animals (Basel) 6, E18.

22. Caporaso JG, Kuczynski J, Stombaugh J, et al. (2010) QIIME allows analysis of high-throughput community sequencing data. Nat Methods 7, 335-336.

23. Edgar RC (2010) Search and clustering orders of magnitude faster than BLAST. Bioinformatics 26, 2460-2461.

24. Edgar RC, Haas BJ, Clemente JC, et al. (2011) UCHIME improves sensitivity and speed of chimera detection. Bioinformatics 27, 2194-2200.

25. Love MI, Huber W \& Anders S (2014) Moderated estimation of fold change and dispersion for RNA-seq data with DESeq 2. Genome Biology 15, 550.

26. Huber W, Carey JV, Gentleman R, et al. (2015) Orchestrating high-throughput genomic analysis with Bioconductor. Nat Methods 12, 115-121.

27. Benjamini Y \& Hochberg Y (1995) Controlling the false discovery rate: a practical and powerful approach to multiple testing. $J$ R Stat Soc Series B Stat Methodol 57, 289-300.

28. Oksanen J, Blanchet FG, Friendly M, et al. (2018) Vegan: Community Ecology Package. R Package Version 2.4-3. https://cran.r-project.org/web/packages/vegan/index.html (accessed October 2018)

29. Lê Cao K-A, Costello M-E, Lakis VA, et al. (2016) MixMC: a multivariate statistical framework to gain insight into microbial communities. PLOS ONE 11, e0160169.

30. Rohart F, Gautier B, Singh A, et al. (2017) mixOmics: an R package for 'omics feature selection and multiple data integration. PLoS Comput Biol 13, e1005752.

31. Kononoff PJ \& Hanford KJ (2006) Technical note: estimating statistical power of mixed models used in dairy nutrition experiments. J Dairy Sci 89, 3968-3971.

32. Regmi PR, Metzler-Zebeli BU, Gänzle MG, et al. (2011) Starch with high amylose content and low in vitro digestibility increases intestinal nutrient flow and microbial fermentation and selectively promotes bifidobacteria in pigs. I Nutr $\mathbf{1 4 1}$, 398-405.

33. Fouhse JM, Gänzle MG, Beattie AD, et al. (2017) Whole-grain starch and fiber composition modifies ileal flow of nutrients and nutrient availability in the hindgut, shifting fecal microbial profiles in pigs. J Nutr 147, 2031-2040.

34. Rashid T, Wilson C \& Ebringer A (2013) The link between ankylosing spondylitis, Crohn's disease, Klebsiella, and starch consumption. Clin Dev Immunol 2013, 872632.

35. Metzler-Zebeli BU, Newman MA \& Zebeli Q (2018) Transglycosylated starch modifies the cecal bacterial metagenome and gene abundance of key catabolic enzymes for bacterial starch metabolism in pigs. In 11th Rowett-INRA Conference - Gut Microbiology. Aberdeen, UK, p. 29.

36. Prins RA (1971) Isolation, culture, and fermentation characteristics of Selenomonas ruminantium var. bryantivar. $n$. from the rumen of sheep. J Bacteriol 105, 820-825.

37. Gänzle MG \& Follador R (2012) Metabolism of oligosaccharides and starch in lactobacilli: a review. Front Microbiol 3, 340.

38. Clavel T, Lepage P \& Charrier F (2014) The family Coriobacteriaceae. In The Prokaryotes, 4th ed., pp. 201-238 [E Rosenberg, EF DeLong, S Lory, E Stackebrandt and F Thompson, editors]. Berlin Heidelberg, Germany: Springer-Verlag.

39. Walter J, Martínez I \& Rose DJ (2013) Holobiont nutrition: considering the role of the gastrointestinal microbiota in the health benefits of whole grains. Gut Microbes 4, 340-346.

40. Carlson JL, Erickson JM, Hess JM, et al. (2017) Prebiotic dietary fiber and gut health: comparing the in vitro fermentations of beta-Glucan, inulin and xylooligosaccharide. Nutrients 9 , E1361.

41. Martínez I, Lattimer JM, Hubach KL, et al. (2013) Gut microbiome composition is linked to whole grain-induced immunological improvements. ISME J 7, 269-280.

42. Flint HJ, Duncan SH, Scott KP, et al. (2015) Links between diet, gut microbiota composition and gut metabolism. Proc Nutr Soc 74, 13-22.

43. Shetty SA, Marathe NP, Lanjekar V, et al. (2013) Comparative genome analysis of Megasphaera sp. reveals niche specialization and its potential role in the human gut. PLOS ONE 8, e79353.

44. Gophna U, Konikoff T \& Nielsen HB (2017) Oscillospira and related bacteria - from metagenomic species to metabolic features. Environ Microbiol 19, 835-841.

45. O'Hea EK \& Leveille GA (1969) Significance of adipose tissue and liver as sites of fatty acid synthesis in pig and efficiency of utilization of various substrates for lipogenesis. J Nutr $\mathbf{9 9}$, 338-344.

46. Morrison DJ \& Preston T (2016) Formation of short chain fatty acids by the gut microbiota and their impact on human metabolism. Gut Microbes 7, 189-200.

47. Windey K, De Preter V \& Verbeke K (2016) Relevance of protein fermentation to gut health. Mol Nutr Food Res 56, 184-196

48. Yao CK, Muir JG \& Gibson PR (2016) Review article: insights into colonic protein fermentation, its modulation and potential health implications. Aliment Pharmacol Ther 43, 181-196.

49. Metzler BU \& Mosenthin R (2008) A review of interactions between dietary fiber and the gastrointestinal microbiota and their consequences on intestinal phosphorus metabolism in growing pigs. Asian-Aust J Anim Sci 21, 603-615. 